Erschienen in: Stickel, Gerhard (Hrsg.): Deutsche Gegenwartssprache. Tendenzen und Perspektiven. - Berlin, New York: de Gruyter, 1990. S. 192-197. (Institut für deutsche Sprache. Jahrbuch 1989)

ROBERT SCHLÄPFER

\title{
Standardsprache und Mundarten in der deutschen Schweiz
}

In einer Darstellung des Verhältnisses zwischen Mundart und Standardsprache in der deutschen Schweiz ist auszugehen vom Tatbestand, daß die Mundart in der deutschen Schweiz die täglich gesprochene Sprache ist, die täglich gesprochene Sprache aller sozialen Schichten in allen Alltagssituationen. So reden die Lehrkräfte außerhalb des eigentlichen Unterrichts mit Schülerinnen und Schülern und unter sich, Professoren mit Studierenden und unter sich, Vorgesetzte und Untergebene in Wirtschaft und Industrie nur Mundart miteinander. In der Familie spricht man ausschließlich Mundart. Das alles gilt ohne Unterschied für die ganze deutsche Schweiz.

Der Gebrauch der gesprochenen Standardsprache ist auf verhältnismäßig wenige, traditionell bestimmte, meist stark formal geprägte Situationen beschränkt: auf den wissenschaftlichen Vortrag, die feierliche Rede, die Predigt, in der Schule auf den eigentlichen Unterricht, im staatlichen Radio und Fernsehen der deutschen und rätoromanischen Schweiz auf bestimmte Sendegefäße und Textsorten. Dazu kommt der Gebrauch der Standardsprache in der Kommunikation mit Gesprächspartnern, die schweizerdeutsche Mundarten nicht verstehen.

Dieser verhältnismäßig enge Verwendungsbereich der gesprochenen Standardsprache ist seit den sechziger Jahren noch mehr eingeengt worden, als Folge einer sog. 'Mundartwelle', mit der die Mundart in Vortrag, Ansprache, Kirche, Unterricht und in den elektronischen Medien in Bereiche vorgedrungen ist, die bis dahin einigermaßen unbestritten der Standardsprache zugestanden hatten. In den meisten kantonalen Parlamenten der deutschen Schweiz wurde bis zum Zweiten Weltkrieg Standard gesprochen; heute ist das die Ausnahme. Die Sendezeit der in Mundart gesprochenen oder moderierten Produktionen hat beim staatlichen Radio und Fernsehen bis in die zweite Hälfte der achtziger Jahre kontinuierlich zugenommen. Die seit einigen Jahren zugelassenen privaten lokalen Radiosender kennen praktisch nur die Mundart, mit Ausnahme der Agenturmeldungen und Nachrichtensendungen, die ihnen in schriftlicher Form zukommen und die dann abgelesen werden.

Die geschriebene Sprache ist im wesentlichen die Domäne der Standardform des Deutschen geblieben. Gemessen am gesamten Schriftum in Literatur, Presse, Geschäftsverkehr haben geschriebene Mundarttexte nicht viel mehr als marginale Bedeutung. In zwei Bereichen läßt sich aber 
doch auch eine Zunahme geschriebener Mundart feststellen: zum einen im persönlichen Schriftverkehr, in der privaten Korrespondenz vor allem junger Leute, und sehr verbreitet auch in gedruckten familiären Anzeigen, in Vermählungs- und Geburtsanzeigen - bemerkenswerterweise nur ganz selten aber in Todesanzeigen; zum anderen treffen wir geschriebene Mundart häufig an in der Werbung, in Zeitungsinseraten und auf Plakaten: in Schlagworten oder in ganzen Sentenzen. Offenbar hält man die Mundart für werbewirksam.

Als gesprochene Sprache hat die Mundart in der deutschen Schweiz mit wenigen Ausnahmen immer eine unangefochten starke Stellung gehabt. Eine Ausnahme machten his zu einem gewissen Grad nur Städte wie Basel und Zürich in den Jahren vor dem Ersten Weltkrieg, als dort der Anteil ausländischer, vor allem reichșdeutscher Wohnbevölkerung zeitweise 20-30\% ausmachte, so daß man damals in Geschäften an der renommierten Zürcher Bahnhofstraße vom Verkaufspersonal hochdeutsch angesprochen worden sein soll, wenn man nicht Zürichdeutsch, sondern eine andere deutschschweizerische Mundart sprach. Das war aber eben Ausnahme. Als der 1845 in Baselland geborene Dichter Carl Spitteler nach Jahren der Hauslehrer-Tätigkeit im Baltikum zu Hause auch im Kreise von Verwandten und Freunden ein norddeutsch-baltisches Hochdeutsch sprach, wurde ihm das sehr übelgenommen.

Der nun seit zwei Jahrzehnten noch weit über den Alltagsgebrauch hinaus ausgedehnte Mundartgebrauch der Deutschschweizer hat ohne Zweifel auf verschiedenen Ebenen seinen Folgen: in bezug auf die schweizerdeutschen Mundarten selber, in der Beziehung des Deutschschweizers zur gesprochenen deutschen Standardsprache und - im viersprachigen Staat - im Verhältnis der deutschen Schweiz zu den anderen Sprachgebieten.

Zur Mundart selber: Seit dem Ausgang des Mittelalters haben sich auf dem Boden der deutschen Schweiz auf vergleichsweise engem Raum zwischen dem Bodensee und der deutsch-französischen Sprachgrenze, zwischen Basel und dem Oberwallis auf Grund der zum Teil topographisch bedingten historisch-politischen und kulturell-konfessionellen Kleinräumigkeit verhältnismäßig große Unterschiede zwischen den regionalen Mundarten herausgebildet. Heute bilden Kantonsgrenzen und Konfessionsunterschiede keine Barrieren mehr, die Mobilität der Bevölkerung und die elektronischen Medien fördern einen innersprachlichen Ausgleich. Diese Tendenz wird ohne $Z$ weifel verstärkt und beschleunigt durch den extensiven Mundartgebrauch, den die letzten Jahre gebracht haben. „Schweizerdeutsch" - das ist im Grunde bis heute ein mehr oder weniger fiktiver Begriff, gab es doch bis dahin keine mundartlichen Erscheinungen, die das ganze Gebiet der deutschen Schweiz abgedeckt und 
sich gleichzeitig nur auf die deutsche Schweiz beschränkt hätten. Heute dürften wir nun aber auf ein Schweizerdeutsch hin zumindest unterwegs sein, in einem doppelten Sinn: einerseits, indem sich die lokalen und regionalen Mundarten innerhalb der deutschen Schweiz stärker aneinander angleichen, und andererseits, weil sich mit diesem Prozeß viele mundartliche Erscheinungen, die bis anhin nur regional verbreitet waren, bis zur Landesgrenze hin ausbreiten, dort aber haltmachen. So entwickelt sich allmählich ein Schweizerdeutsch, das aber immer noch unüberhörbare regionale Varianten aufweist und immer noch unzweifelhaft Mundart ist und keineswegs eine irgendwo zwischen Mundart und Standardsprache angesiedelte Umgangssprache, wie auch immer man "Umgangssprache" genauer definieren will.

Dann die Beziehung der Deutschschweizerinnen und Deutschschweizer zur gesprochenen Standardsprache: Diese Beziehung war seit jeher eine schwierige Sache, seit man in der deutschen Schweiz begonnen hatte, die von außen übernommene Schriftsprache auch zu sprechen. Und die von verschiedenen Seiten her immer wieder genährte Vorstellung von der von allen landschaftlichen Schlacken befreiten "reinen deutschen Hochsprache" hat den Deutschschweizern stets zu schaffen gemacht. Wilhelm Wackernagel, nach dem Tode Jakob Grimms im Jahre 1863 wohl einer der bedeutendsten Germanisten seiner Zeit, war 1833 von Berlin nach Basel gekommen. Hier glaubte er, unermüdlich vor allen schriftsprachfremden Einflüssen auf die Hochsprache als "Provinzialismen, Barbarismen, Archaismen" eindringlich warnen zu müssen. Als Hochschullehrer hat er mit dieser Vorstellung das Sprachbewußtsein von Germanisten, Lehrern, Theologen bis weit ins 20 . Jahrhundert hinein geprägt. Noch heute sind viele Deutschschweizer überzeugt, ein Standarddeutsch sei ein objektiv besseres Deutsch, je nördlicher es töne. Das führt natürlich unweigerlich zu deutschschweizerischen Minderwertigkeitsgefühlen. Andererseits haben in unserem Jahrhundert die politischen Verhältnisse des Dritten Reiches und der damalige großdeutsche Machtanspruch bei vielen Deutschschweizern zu einer tiefgehenden emotionalen Aversion gegen die gesprochene Standardsprache geführt.

Wie ambivalent in der deutschen Schweiz das Verhältnis zur gesprochenen Standardsprache ist, zeigt sich exemplarisch etwa darin, wie die Sprache der Deutschschweizer Radio- und Fernseh-Sprecher und Sprecherinnen immer wieder Gegenstand der Kritik ist. Seit es ein Schweizer Radio gibt, sind zu keinem anderen Thema so viele Leserbriefe geschrieben worden: Wenn eine Sprecherin ein tadelloses Bühnendeutsch spricht, heißt es, wir brauchten doch nicht dieses Deutschtum und Deutsch-Tun in unserem Radio; läßt sich aber ein Moderator mit einer gemäßig- 
ten schweizerischen Variante der Standardsprache hören, ist die andere Seite empört: Nun können nicht einmal mehr die Berufssprecher richtig Deutsch!

Auch in der Schule tut man sich vielfach schwer mit der deutschen Standardsprache, gesprochen und geschrieben. Und hat dann sehr rasch die immer wieder kolportierte Entschuldigung zur Hand, die Standardsprache sei eben für Deutschschweizerinnen und Deutschschweizer eine Fremdsprache. Daß das linguistisch nicht zutrifft, brauchen wir hier nicht zu erörtern. Aber es ist das tatsächlich, wie es Horst Sitta recht treffend formuliert, so etwas wie eine psychische Realität. Störend und widersprüchlich an der Behauptung von der Standardsprache als Fremdsprache ist dann aber, daß sie von vielen Lehrkräften zwar immer wieder vorgebracht wird, daß jedoch niemand die didaktisch-methodischen Konsequenzen aus der Behauptung zieht und den Versuch macht, die "Fremdsprache Standarddeutsch" wie die Fremdsprachen Englisch und Französisch zu lehren und lernen zu lassen.

$\mathrm{DaB}$ die Standardsprache in jüngster Zeit noch weniger gesprochen wird, aus traditionellen Verwendungsbereichen durch die Mundart verdrängt, führt natürlich zu einem Teufelskreis. Mangels Übung fehlt es an einer gewissen Gewandtheit, was unter den geschilderten Umständen die Unsicherheit und eine womöglich latent vorhandene, unterschwellige innere Ablehnung verstärkt: Man nützt die Möglichkeiten, die Sprache zu sprechen, nicht und weicht der Notwendigkeit, sie zu brauchen, wenn möglich aus. Berner Gymnasiasten, in einer repräsentativen Untersuchung befragt, welche Sprache sie vorziehen - Standarddeutsch, Englisch oder Französisch - in der Kommunikation mit einem Gesprächspartner, der Schweizerdeutsch nicht versteht, haben mit einer Mehrheit von über $60 \%$ erklärt, dem Englischen den Vorzug zu geben. Ob sie es dann in der Praxis wirklich so halten, ist eine andere Frage. Daß sie so antworten, sagt aber immerhin einiges aus über ihre Einstellung oder ihre Vorurteile.

Und nun der dritte Problemkreis: das Verhältnis von Mundart und Standardsprache in der deutschen Schweiz in seinen Auswirkungen auf die Beziehungen zu den anderen Sprachregionen in der mehrsprachigen Schweiz. Wenn wir die Ausbreitung der Mundart auf Kosten der Standardsprache in praktisch allen Bereichen der gesprochenen Sprache geschildert haben, müssen wir jetzt eine Einschränkung machen: Im Plenum und in den Kommissionen der eidgenössischen Räte - des Ständerates und des Nationalrates - wie auch in der Bundesverwaltung hat man sich immer strikt daran gehalten und hält sich daran, daB man konsequent Standard spricht, wenn nur eine einzige französisch- oder italienischsprachige Persönlichkeit zugegen ist. In diesen Kreisen gehört 
es zu den unumstößlichen politischen Spielregeln, daß die Mehrheit auf die Minderheiten Rücksicht nimmt.

Anders in politisch weniger sensibilisierten Kreisen, in mehrsprachigen Wirtschafts-, Verbands-, Vereinsgremien, in privaten Zirkeln. Hier fällt es den Deutschschweizerinnen und Deutschschweizern zunehmend schwerer, sich gegebenenfalls ohne Einschränkung an die Standardsprache zu halten. In einer Kommission von sieben Mitgliedern - sechs deutscher und eines französischer Muttersprache - wendet man sich im Standard oder unter Umständen französisch an den welschen Gesprächspartner. Und auch unter sich spricht man - aus Rücksicht - Standarddeutsch, mindestens eine Weile lang. Irgendwie empfindet man es aber als unnatürlich, und mit zunehmender Ermüdung immer unnatürlicher. Bis dann der eine oder andere mit einem ersten Mundart-Einsprengsel aus der anfänglichen Rücksicht ausbricht. Wohlverstanden: nicht gegen den Romand selber, aber in dessen Gegenwart unter den Deutschschweizern, womit jener doch aus einem Teil der Kommunikation ausgeschlossen wird.

Der überbordende Mundartgebrauch der Deutschschweizer wird in der französischen und in der italienischen Schweiz mit zunehmender Verbitterung registriert. Nicht so virulent ist das Problem in der Beziehung zur vierten Landessprache, zum Rätoromanischen, weil es kaum mehr Rätoromanen gibt, die nicht auch Bündnerdeutsch sprechen. Die Verärgerung der italienischen und französischen Landesteile dagegen läßt sich der Presse entnehmen: häufigen redaktionellen Äußerungen, aber auch Leserbriefen und kulturpolitischen Artikeln. Sie kommt in sprachpolitischen Diskussionen in den elektronischen Medien zur Sprache. Man redet immer wieder davon, der Graben zwischen der welschen und der deutschen Schweiz werde zunehmend tiefer.

Von einem solchen Graben war erstmals in den Vorkriegs- und Kriegsjahren des Ersten Weltkriegs die Rede gewesen, als man in der deutschen Schweiz bis hinauf in die oberste Armeeführung seinen Sympathien für die Mittelmächte, insbesondere für das wilhelminische Deutschland unverhohlen Ausdruck gab. Es war damals Carl Spitteler, der mit seiner denkwürdigen Rede "Unser Schweizer Standpunkt” am 14. Dezember 1914 die Deutschschweizer zur Besonnenheit aufrief. Das brachte ihm - und vielen anderen einsichtigen Deutschschweizern - den tiefen Dank der Suisse romande, in Deutschland aber das völlige Totschweigen seines dort verlegten literarischen Werks. 
Vom Graben zwischen Deutschschweiz und Westschweiz war dann lange nicht mehr die Rede. Als man in den dreißiger Jahren und in den ersten Jahren des Zweiten Weltkriegs in der Deutschschweiz im Zuge der sog. "geistigen Landesverteidigung" gegenüber Deutschland immer stärker die eigene Mundartlichkeit betonte, begegnete man dieser Bewegung in der französischen Schweiz mit Verständnis, ja sogar mit einem gewissen Wohlwollen, sah man doch darin die bewufte Distanzierung der Deutschschweizer vom Reich und das Bekenntnis zur eigenen Nation, die eben keine Sprachnation ist.

Doch heute scheint der Graben wieder aufgerissen zu werden. Als Folge der deutschschweizerischen Mundartwelle, oder zumindest angeblich wegen der Mundartwelle. Man fragt sich in der französischen Schweiz, wozu man denn Deutsch, Standarddeutsch lerne, wenn man es doch in der deutschen Schweiz kaum brauchen könne. Und man schickt seine jungen Leute zunehmend eher in die Bundesrepublik zum Sprachaufenthalt. Dabei müssen wir uns bewußt sein, daß sich an diesem scheinbar rein sprachlichen oder zumindest scheinbar vor allem sprachlichen Problem letztlich ein ganz anderes Unbehagen kristallisiert: das Gefühl nämlich der Bedrohung der Minderheit durch das große politische Gewicht der deutschen Schweiz und vor allem durch das Gewicht der deutschschweizer Wirtschaftsmacht auch in der romanischen Schweiz. Sprachprobleme sind hier im Grunde wie an anderen Orten nicht nur - oder vielleicht sogar nur am Rande - Sprachprobleme.

In der deutschen Schweiz ist es aber in der gegenwärtigen Situation gar nicht so leicht, die Heranwachsenden davon zu überzeugen, daß sie die deutsche Standardsprache nicht nur lesen und verstehen, sondern auch sprechen und schreiben sollten. Sie sehen es schwer ein, wozu das nötig ist - im Gegensatz zum Interesse am Englischen. In der Institution Schule versucht man, dem Problem mit Verordnungen und Reglementen beizukommen. Aber Verordnungen und Reglemente geben keine gute Lernmotivation ab. Und auch kulturpolitische Argumente im mehrsprachigen Staat regen Schüler kaum zu geduldigem Lernen an. So müssen wir immer wieder auf ganz verschiedenen Ebenen zu überzeugen versuchen: daß Dialekt und Standard zusammengehören und sich ergänzen, von der Nähe zur Weite. Daß wir als Deutschschweizer auch in Deutschland und in Österreich sollten erzählen, argumentieren, überreden und widersprechen können - nicht nur dort, wo man Schweizerdeutsch spricht oder Englisch. 